\title{
Effect of luteal phase support with vaginal progesterone on pregnancy outcomes in natural frozen embryo transfer cycles: A meta-analysis
}

\author{
Aeran Seol ${ }^{1, *}$, Yoo Jin Shim ${ }^{2,3, *}$, Sung Woo Kim ${ }^{1}$, Seul Ki Kim ${ }^{2,3}$, Jung Ryeol Lee ${ }^{2,3}$, Byung Chul Jee ${ }^{2,3}$, Chang Suk Suh ${ }^{1,3}$, \\ Seok Hyun Kim ${ }^{1,3}$ \\ ${ }^{1}$ Department of Obstetrics and Gynecology, Seoul National University Hospital, Seoul; ${ }^{2}$ Department of Obstetrics and Gynecology, Seoul National \\ University Bundang Hospital, Seongnam; ${ }^{3}$ Department of Obstetrics and Gynecology, Seoul National University College of Medicine, Seoul, Korea
}

Objective: The purpose of this study was to determine the effect of vaginal progesterone for luteal phase support (LPS) on the clinical pregnancy rate (CPR) in natural frozen embryo transfer (FET) cycles via a meta-analysis.

Methods: We performed a meta-analysis of randomized controlled trials (RCTs) and retrospective studies that met our selection criteria. Four online databases (PubMed, Embase, Medline, and the Cochrane Library) were searched between January 2017 and May 2017. Studies were selected according to predefined inclusion criteria and meta-analyzed using $R$ software version 2.14.2. The main outcome measure was CPR.

Results: A total of 18 studies were reviewed and assessed for eligibility. One RCT $(n=435)$ and three retrospective studies $(n=3,033)$ met the selection criteria. In a meta-analysis of the selected studies, we found no significant difference in the CPR (odds ratio [OR], $0.96 ; 95 \%$ confidence interval [Cl], 0.60-1.55) between the vaginal progesterone and control groups. An analysis of the two retrospective cohort studies that reported the live birth rate (LBR) following FET showed a significantly higher LBR in the vaginal progesterone group (OR, 1.72; 95\% Cl, 1.21-2.46). A subgroup meta-analysis of FET conducted 5 days after injection of human chorionic gonadotropin showed no significant differences between the two groups with regard to the $\mathrm{CPR}(\mathrm{OR}, 1.18 ; 95 \% \mathrm{Cl}, 0.90-1.55)$ or miscarriage rate $(\mathrm{OR}, 0.73 ; 95 \% \mathrm{Cl}, 0.36-1.47)$.

Conclusion: The results of this meta-analysis of the currently available literature suggest that LPS with vaginal progesterone in natural FET cycles does not improve the CPR.

Keywords: Embryo transfer; Fertilization in vitro; Luteal phase; Pregnancy; Progesterone

\section{Introduction}

Progesterone is an essential hormone for embryonic implantation and the maintenance of pregnancy [1]. Progesterone generated by the corpus luteum prepares the endometrium for the implantation

Received: July 1, 2019· Revised: October 30, 2019. Accepted: November 11, 2019 Corresponding author: Seul Ki Kim

Department of Obstetrics and Gynecology, Seoul National University Bundang Hospital, 82 Gumi-ro 173beon-gil, Bundang-gu, Seongnam 13620, Korea Tel:+82-31-787-7264 Fax:+82-31-787-4054 E-mail:drksk80@gmail.com

*These authors contributed equally to this work.

This is an Open Access article distributed under the terms of the Creative Commons Attribution Non-Commercial License (http://creativecommons.org/licenses/by-nc/4.0/) which permits unrestricted non-commercial use, distribution, and reproduction in any medium, provided the original work is properly cited. of the embryo. Additionally, progesterone protects the embryo by regulating the immune system to facilitate the production of noninflammatory Th2 cytokines [2]. During early pregnancy, inadequate production of progesterone can result in a decreased probability of clinical pregnancy, an increased probability of miscarriage, and a decreased likelihood of live birth [3].

Several routes of administration exist for progesterone, including oral, intramuscular, and vaginal progesterone. Oral progesterone is rapidly metabolized. The vaginal and intramuscular routes of administration of micronized progesterone have higher bioavailability than oral administration [4]. Vaginal administration was shown to induce sufficient endometrial development; as such, it is preferable to other routes of administration because the vaginal route is less painful and 
easier to apply than intramuscular injections [5,6].

Various protocols for frozen embryo transfer (FET) exist depending on the method of endometrial preparation. Natural-cycle FET is a method that does not involve hormone exposure for endometrial preparation, while artificial-cycle FET involves priming the endometrium with a combination of exogenous estrogen and progesterone. These two methods have commonly been used in women with regular ovulatory menstrual cycles. Few studies have been conducted on the effect of luteal-phase progesterone supplementation as part of the natural FET cycle on the pregnancy rate. The first study that used vaginal progesterone as luteal phase support (LPS) in naturalcycle FET was published in 2010 [7], and other studies have subsequently been conducted [8-10]. However, the results have been inconsistent and controversial. In light of the above considerations, we performed a meta-analysis to compare the pregnancy outcomes in women treated with vaginal progesterone with those of women administered a placebo at the time of embryo transfer (ET).

\section{Methods}

We searched four online databases (PubMed, Embase, Medline, and the Cochrane Library) for all relevant randomized controlled trials (RCTs) and retrospective studies under the following Medical Sub- ject Heading terms to generate subsets of studies: (1) "frozen embryo transfer" or "FET" or "cryopreservation" or "frozen-thawed embryo", (2) "progesterone" or "luteal phase support", and (3) "pregnancy" or "pregnancy rate" or "live birth" or "implantation" or "embryo transfer" (with a subset of 1 and either 2 or 3 ) with "AND” to identify citations appropriate for evaluating the effect of vaginal progesterone for LPS on the clinical pregnancy rate (CPR). The databases were searched between January 2017 and May 2017 without restriction by country, sample size, or blinding. The searches were independently conducted by two reviewers (AS and SWK).

The target population was infertile women with normal ovulatory menstrual cycles who were undergoing FET. Patients in the experimental group were supplemented with vaginal progesterone for LPS, whereas no luteal support was used in the control group. The primary outcome was the CPR. All manuscripts were independently reviewed in full for the selection and exclusion of publications according to predefined inclusion criteria. The extraction of data from each study (e.g., information such as the study design, sample size, and outcomes) was also independently conducted by the authors of the present study using predetermined tables and forms. Disagreements regarding article selection or data extraction were resolved via consensus or arbitration by a third reviewer. The risk of bias was illustrated as a risk-of-bias graph. The evidence was summarized using a

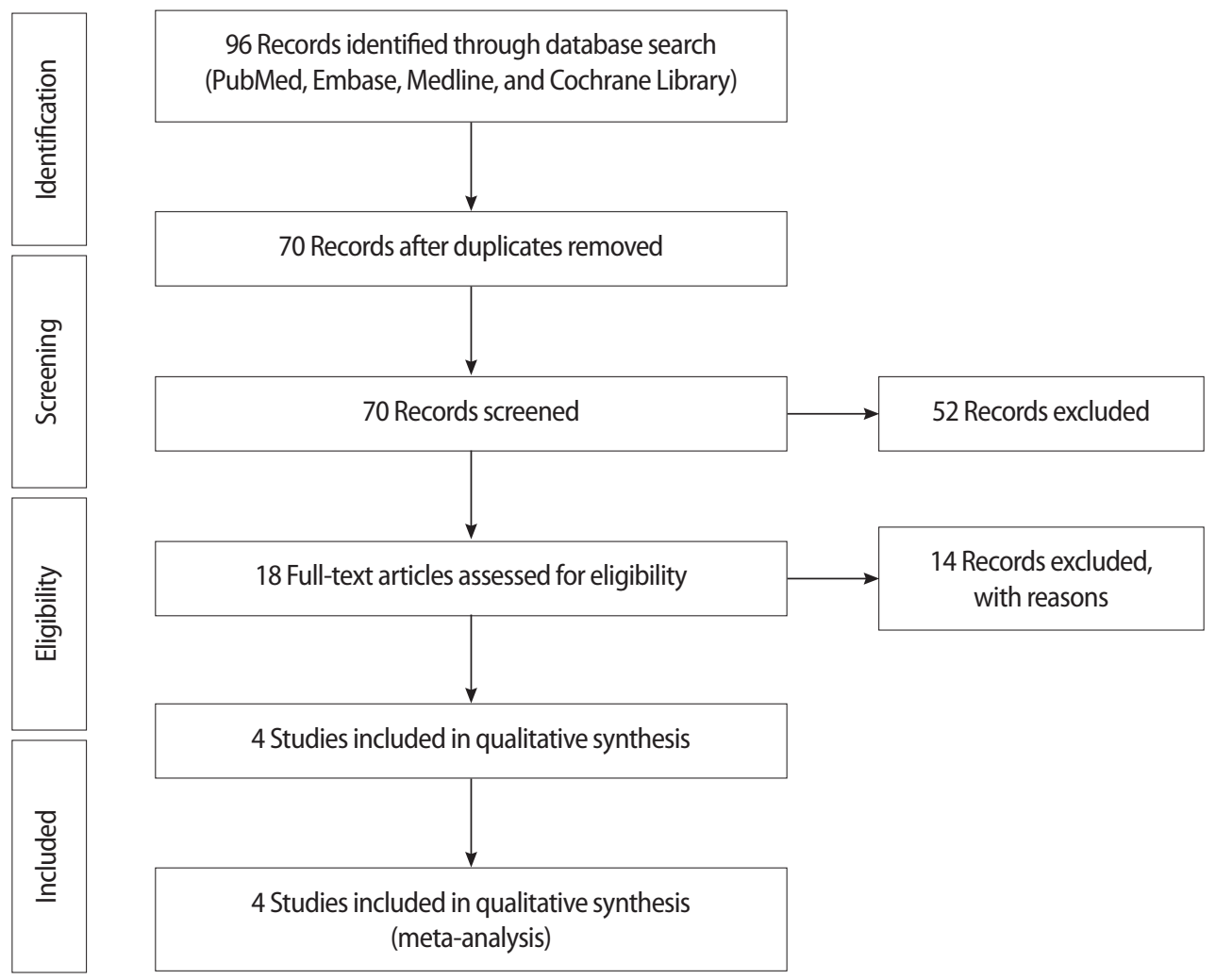

Figure 1. Flow diagram of the selected studies. 
tool provided by the Cochrane Collaboration.

We used R software ver. 2.14.2 (R Foundation, Vienna, Austria) for meta-analysis. Odds ratios (ORs) with 95\% confidence intervals (Cls) were calculated using the Mantel-Haenszel method for binary variables. The heterogeneity of treatment effects was graphically assessed using forest plots and evaluated using the $\mathrm{I}^{2}$ statistic, and heterogeneity was considered absent if $\mathrm{I}^{2}<50 \%$. The meta-analysis, including the results of the subgroup analysis, was displayed in the form of forest plots and an adjusted funnel plot.

\section{Results}

The database search yielded 96 publications, 70 of which were reviewed after the exclusion of duplicates. After the initial selection process, 18 full-text articles were assessed for eligibility. One RCT $(n=435)[9]$ and three retrospective studies $(n=3,033)[7,8,10]$ met the inclusion criteria. The Preferred Reporting Items for Systematic Reviews and Meta-Analyses flowchart explaining the study selection process for this meta-analysis is shown in Figure 1.

The RCT evaluated in this meta-analysis randomly allocated subjects to the experimental group $(n=219)$ or the control group $(n=216)$ to analyze the effect of progesterone support. Subjects in the experimental group were administered $400 \mathrm{mg}$ of vaginal progesterone twice a day. In total, the three retrospective cohort studies assessed 3,033 women, of whom 2,240 women were allocated to the experimental group of their respective study, while 793 women were allocated to the control group. The characteristics of the selected studies are shown in Table 1. In two of the four studies, $200 \mathrm{mg}$ of micronized vaginal progesterone (Utrogestan) was administered three times daily. In the remaining two studies, the study group was administered $90 \mathrm{mg}$ of vaginal gel (Crinone gel, 8\%) daily. In three of the four studies, ET was performed 5 days after human chorionic gonadotro-

Table 1. Characteristics of the included studies

\begin{tabular}{|c|c|c|c|c|c|c|c|}
\hline Study & $\begin{array}{l}\text { No. of } \\
\text { subjects }\end{array}$ & Design & Inclusion criteria & Intervention group & Control group & Timing of ET & Outcome \\
\hline $\begin{array}{l}\text { Bjuresten et al. } \\
\text { (2011) [9] }\end{array}$ & 435 & $\begin{array}{c}\text { Prospective } \\
\text { RCT }\end{array}$ & $\begin{array}{l}\text { Women with unexplained } \\
\text { infertility, tubal factor } \\
\text { infertility, or male factor } \\
\text { infertility }\end{array}$ & $\begin{array}{l}\text { Vaginal micronized } \\
\text { progesterone, } \\
400 \text { mg twice } \\
\text { daily }\end{array}$ & $\begin{array}{c}\text { No } \\
\text { progesterone }\end{array}$ & $\begin{array}{l}3 \text { Days after the LH } \\
\text { surge ( } 5 \text { days after } \\
\text { hCG injection) }\end{array}$ & $\begin{array}{l}\text { Pregnancy test results, early } \\
\text { miscarriage rate, clinical } \\
\text { pregnancy rate, spontaneous } \\
\text { abortion, and live birth rate }\end{array}$ \\
\hline $\begin{array}{l}\text { Kim et al. } \\
\text { (2014) [8] }\end{array}$ & 228 & Retrospective & $\begin{array}{l}\text { Maternal age between } 20 \\
\text { and } 41 \text { years, natural } \\
\text { ovulatory cycles of } 24 \text { to } \\
35 \text { days in length, and } \\
\text { body mass index } \\
\text { between } 18 \text { and } 25 \mathrm{~kg} / \mathrm{m}^{2}\end{array}$ & $\begin{array}{l}\text { Vaginal } \\
\text { progesterone gel, } \\
90 \text { mg once daily }\end{array}$ & $\begin{array}{c}\text { No } \\
\text { progesterone }\end{array}$ & $\begin{array}{l}5 \text { Days after hCG } \\
\text { injection }\end{array}$ & $\begin{array}{l}\text { Clinical pregnancy rate, } \\
\text { miscarriage rate, embryo } \\
\text { implantation rate, and } \\
\text { live birth rate }\end{array}$ \\
\hline $\begin{array}{l}\text { Montagut et al. } \\
\text { (2016) [10] }\end{array}$ & 2,353 & Retrospective & $\begin{array}{l}\text { Maternal age between } 18 \\
\text { and } 39 \text { years old at the } \\
\text { time of oocyte retrieval }\end{array}$ & $\begin{array}{l}\text { Vaginal micronized } \\
\text { progesterone, } \\
200 \text { mg three times } \\
\text { daily }\end{array}$ & $\begin{array}{l}\text { No } \\
\text { progesterone }\end{array}$ & $\begin{array}{l}\text { 3-Day ET or } \\
\text { 5-day ET }\end{array}$ & Clinical pregnancy rate \\
\hline $\begin{array}{r}\text { Kyrou et al. } \\
(2010) \text { [7] }\end{array}$ & 452 & Retrospective & $\begin{array}{l}\text { Maternal age } \leq 37 \text { years } \\
\text { and regular menstrual } \\
\text { cycle }\end{array}$ & $\begin{array}{l}\text { Vaginal micronized } \\
\text { progesterone, } \\
200 \text { mg three times } \\
\text { daily }\end{array}$ & $\begin{array}{l}\text { No } \\
\text { progesterone }\end{array}$ & $\begin{array}{l}5 \text { Days after hCG } \\
\text { injection }\end{array}$ & $\begin{array}{l}\text { Miscarriage rate, implantation } \\
\text { rate, and ongoing pregnancy } \\
\text { rate }\end{array}$ \\
\hline
\end{tabular}

ET, embryo transfer; RCT, randomized controlled trial; LH, luteinizing hormone; hCG, human chorionic gonadotropin.

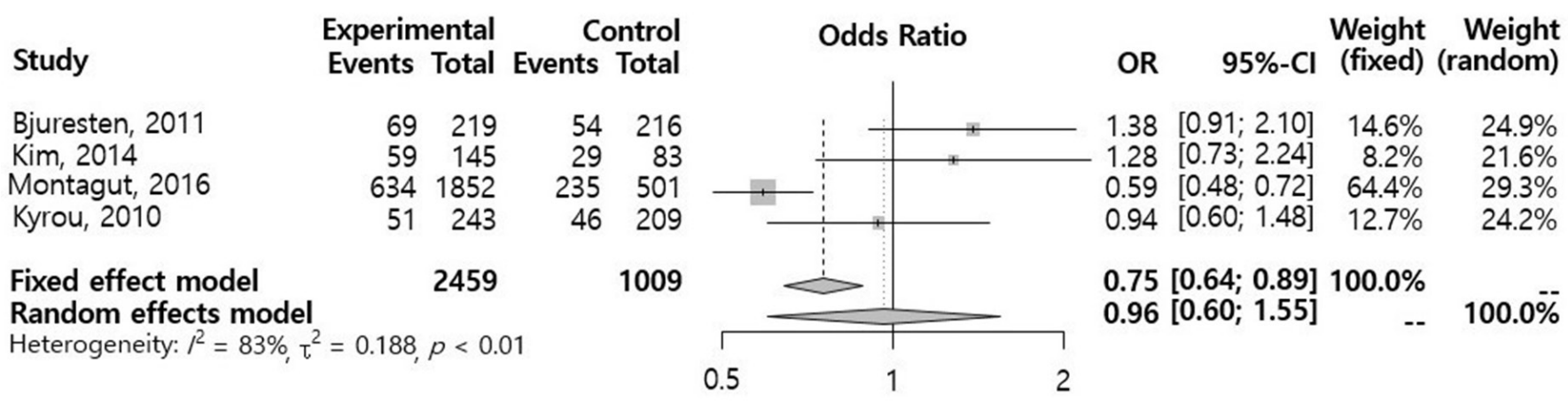

Figure 2. Meta-analysis of the clinical pregnancy rate. $\mathrm{OR}$, odd ratio; $\mathrm{Cl}$, confidence interval. 


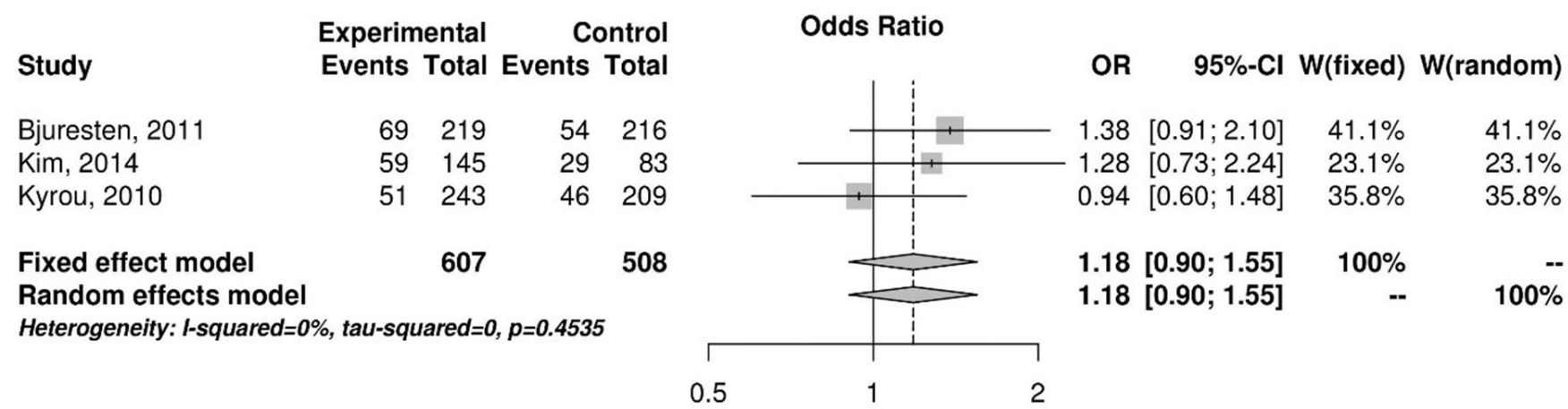

Figure 3. Subgroup analysis of the clinical pregnancy rate (5 days after human chorionic gonadotropin injection). $\mathrm{OR}$, odds ratio; $\mathrm{Cl}$, confidence interval.

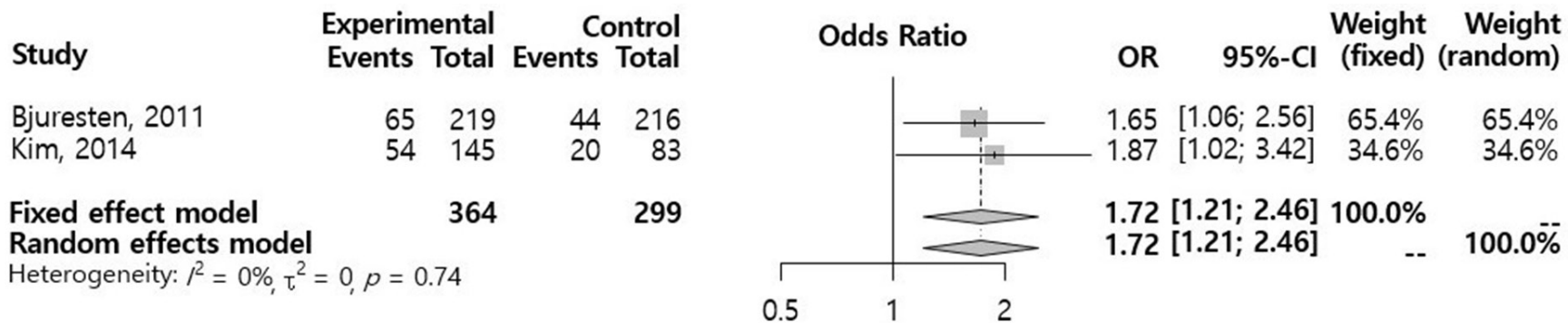

Figure 4. Meta-analysis of the live birth rate. $\mathrm{OR}$, odds ratio; $\mathrm{Cl}$, confidence interval.

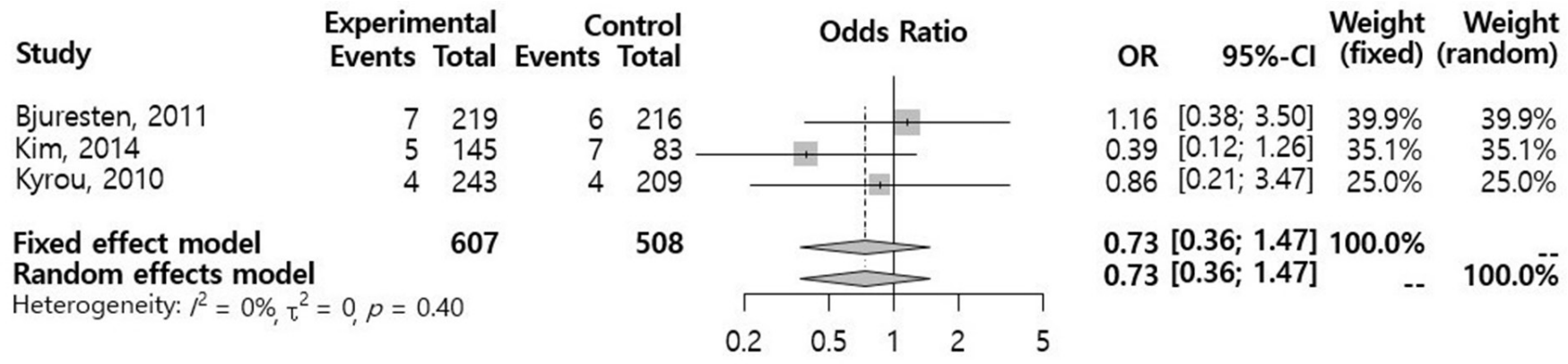

Figure 5. Meta-analysis of the miscarriage rate. $\mathrm{OR}$, odds ratio; $\mathrm{Cl}$, confidence interval.

pin (hCG) injection. In the remaining study, 3-day ET was performed 5 days after hCG injection or 4 days after the luteinizing hormone surge, and 4-day ET was applied 6 days after hCG injection or 5 days after the luteinizing hormone surge.

In a meta-analysis using a random effects model for the selected studies, no significant differences were observed in the CPR (OR, 0.96; $95 \% \mathrm{Cl}, 0.60-1.55$ ) (Figure 2). A subgroup meta-analysis regarding ET performed 5 days after hCG injection also showed no significant difference in the $\mathrm{CPR}(\mathrm{OR}, 1.18 ; 95 \% \mathrm{Cl}, 0.90-1.55)$ (Figure 3$)$. An analysis of two retrospective cohort studies that reported the live birth rate (LBR) following FET showed a significantly higher LBR in the vaginal progesterone group $(\mathrm{OR}, 1.72 ; 95 \% \mathrm{Cl}, 1.21-2.46)$ (Figure 4). The miscarriage rate (MR) was not significantly different between the experi- mental group and the control group in this subgroup analysis (OR, $0.73 ; 95 \% \mathrm{Cl}, 0.36-1.47$ ) (Figure 5).

\section{Discussion}

Our meta-analysis examined the effect of vaginal administration of progesterone on pregnancy outcomes of FET in normal ovulatory women. Three of four studies demonstrated that vaginal progesterone had no beneficial effect on pregnancy outcomes [7-9], while one study showed a negative effect on the CPR [10]. The results of the meta-analysis of these four studies suggest that LPS with vaginal progesterone does not improve the CPR in natural FET cycles.

It is important to keep in mind that the subjects of this study were 
normal ovulatory women. There is abundant evidence that insufficient progesterone secretion at the time of implantation or during early pregnancy is a cause of recurrent implantation failure, sporadic and recurrent miscarriage, and bleeding in early pregnancy. The effects of vaginal progesterone supplementation on the CPR in the subgroups of patients with a history of repeated miscarriage, recurrent implantation failure, or a bleeding event during early pregnancy require additional studies for validation due to the contradictory results of previous studies. Clearly, however, clinicians should pay special attention to LPS for these subgroups of infertile patients.

Our subgroup meta-analysis included three studies in which FET in all subjects was performed 5 days after the hCG injection. Bjuresten et al. [9] showed that progesterone supplementation after ET had no positive effect on the CPR, but improved the LBR (LBR in the progesterone group vs. the control group, $30 \%$ vs. $20 \%$, respectively; $p=0.027$ ). In that study, no significant difference was found in the MR between the two groups. Kim et al. [8] noted that luteal-phase progesterone administration decreased the MR (MR in the progesterone group vs. the control group, $8.5 \%$ vs. $24.1 \%$, respectively; $p=0.044$ ), but no significant difference in the CPR was observed. Meta-analysis of the three groups in which FET was performed 5 days after the hCG injection also demonstrated no statistically significant difference in the CPR.

Our meta-analysis of two retrospective studies (Bjuresten et al. [9] and Kim et al. [8]) yielded a significantly higher LBR in the vaginal progesterone group. However, no significant difference was observed regarding the CPR. While no significant difference was seen with respect to the MR, the patients administered vaginal progesterone displayed a lower MR than the control patients. These results suggest that an increase in the LBR would be observed due to the decrease in the MR, even if no difference in the CPR was present. This result of our study suggests an interesting hypothesis regarding the capacity of vaginal progesterone administration to increase the LBR by diminishing the MR. However, due to the lack of statistical significance regarding the decreased MR in the progesterone group, more studies are required to clarify the effects of vaginal progesterone on LBR, CPR, and MR. Moreover, further large-scale studies on obstetric outcomes including MR and preterm birth are needed to elucidate the explanation for the higher LBR despite the lack of significant difference in the CPR associated with vaginal administration of progesterone.

The methods of the selected studies varied, especially with respect to the dose of vaginal progesterone. Bjuresten et al. [9] used $400 \mathrm{mg}$ of vaginal micronized progesterone administered twice per day, while Kim et al. [8] applied $90 \mathrm{mg}$ of vaginal progesterone gel once daily. Montagut et al. [10] administered $200 \mathrm{mg}$ of vaginal micronized progesterone three times per day. These differences in progesterone dose may have resulted in different outcomes with respect to the CPR.
The major limitation of our study is that it included only one RCT. Due to the paucity of existing data, this inclusion of only one RCT was inevitable. Since the purpose of our study was to investigate the effect of LPS in the form of vaginal progesterone on the CPR in FET cycles via a meta-analysis, we included relevant studies irrespective of the study design. In conclusion, this meta-analysis demonstrated that vaginal progesterone supplementation does not affect the CPR in FET in infertile women with normal ovulatory cycles. Future largescale, prospective, randomized studies are necessary to confirm these findings.

\section{Conflict of interest}

Byung Chul Jee has been an editor of Journal of Clinical and Experimental Reproductive Medicine since 2018; however, he was not involved in the peer reviewer selection, evaluation, or decision process of this article. No other potential conflicts of interest relevant to this article were reported.

\section{ORCID}

Aeran Seol https://orcid.org/0000-0003-4113-2280

Yoo Jin Shim https://orcid.org/0000-0002-6895-3321

Sung Woo Kim https://orcid.org/0000-0003-4689-1323

Seul Ki Kim https://orcid.org/0000-0002-1647-6711

Jung Ryeol Lee https://orcid.org/0000-0003-3743-2934

Byung Chul Jee https://orcid.org/0000-0003-2289-6090

Chang Suk Suh https://orcid.org/0000-0003-1835-7350

Seok Hyun Kim https://orcid.org/0000-0003-0649-3224

\section{Author contributions}

Conceptualization: SKK. Data curation: SKK. Formal analysis: SKK, AS. Methodology: SWK, SKK, JRL, BCJ, CSS, SHK. Project administration: SWK, SKK, JRL, BCJ, CSS, SHK. Visualiuzation: AS, YJS, SKK, JRL, BCJ. Writing-original draft: AS, SKK. Writing-review \& editing: YJS, SKK.

\section{References}

1. Csapo Al, Pulkkinen MO, Ruttner B, Sauvage JP, Wiest WG. The significance of the human corpus luteum in pregnancy maintenance. I. Preliminary studies. Am J Obstet Gynecol 1972;112:1061-7.

2. Szekeres-Bartho J, Wilczynski JR, Basta P, Kalinka J. Role of progesterone and progestin therapy in threatened abortion and preterm labour. Front Biosci 2008;13:1981-90.

3. Daya S. Luteal support: progestogens for pregnancy protection. 
Maturitas 2009;65 Suppl 1:S29-34.

4. Kimzey LM, Gumowski J, Merriam GR, Grimes GJ Jr, Nelson LM. Absorption of micronized progesterone from a nonliquefying vaginal cream. Fertil Steril 1991;56:995-6.

5. Khan N, Richter KS, Newsome TL, Blake EJ, Yankov VI. Matchedsamples comparison of intramuscular versus vaginal progesterone for luteal phase support after in vitro fertilization and embryo transfer. Fertil Steril 2009;91:2445-50.

6. Salat-Baroux J, Cornet D, Alvarez S, Antoine JM, Tibi C, Mandelbaum J, et al. Pregnancies after replacement of frozen-thawed embryos in a donation program. Fertil Steril 1988;49:817-21.

7. Kyrou D, Fatemi HM, Popovic-Todorovic B, Van den Abbeel E, Camus M, Devroey P. Vaginal progesterone supplementation has no effect on ongoing pregnancy rate in hCG-induced natural frozen-thawed embryo transfer cycles. Eur J Obstet Gynecol Reprod Biol 2010;150:175-9.

8. Kim CH, Lee YJ, Lee KH, Kwon SK, Kim SH, Chae HD, et al. The effect of luteal phase progesterone supplementation on natural frozen-thawed embryo transfer cycles. Obstet Gynecol Sci 2014; 57:291-6.

9. Bjuresten K, Landgren BM, Hovatta O, Stavreus-Evers A. Luteal phase progesterone increases live birth rate after frozen embryo transfer. Fertil Steril 2011;95:534-7.

10. Montagut M, Santos-Ribeiro S, De Vos M, Polyzos NP, Drakopoulos $\mathrm{P}$, Mackens $\mathrm{S}$, et al. Frozen-thawed embryo transfers in natural cycles with spontaneous or induced ovulation: the search for the best protocol continues. Hum Reprod 2016;31:2803-10. 